\title{
An exaggerated sensory component of the gastrocolonic response in patients with irritable bowel syndrome
}

\author{
M Simrén, H Abrahamsson, E S Björnsson
}

\begin{abstract}
Backgroundlaims-Visceral hypersensitivity is a feature of the irritable bowel syndrome (IBS). Postprandial symptoms are common in these patients. The effects of nutrients on colonic perception in IBS are incompletely understood.

Subjects-We studied 13 healthy subjects and 16 patients with IBS-eight had diarrhoea predominant (IBS-D) and eight constipation predominant (IBS-C) IBS.

Methods-Colonic perception thresholds to balloon distension and viscerosomatic referral pattern were assessed before and after duodenal infusion of lipid or saline, respectively. At the end of the infusions, plasma levels of gastrointestinal peptides were determined.
\end{abstract}

Results-Lipids lowered the thresholds for first sensation, gas, discomfort, and pain in the IBS group but only for gas in the control group. The percent reduction in thresholds for gas and pain after lipids was greater in the IBS and IBS-D groups but not in the IBS-C group compared with controls. IBS patients had an increased area of referred discomfort and pain after lipids compared with before infusion whereas the referral area remained unchanged in controls. No group differences in colonic tone or compliance were observed. In both groups higher levels of cholecystokinin, pancreatic polypeptide, peptide YY, vasoactive intestinal polypeptide, and neuropeptide $Y$ were seen after lipids. Motilin levels were higher in patients and differences in the subgroups were observed. Levels of corticotrophin releasing factor were lower in the constipated group than in the diarrhoea group. Conclusions-Postprandial symptoms in IBS patients may be explained in part by a nutrient dependent exaggerated sensory component of the gastrocolonic response. (Gut 2001;48:20-27)

Keywords: irritable bowel syndrome; lipids; colonic perception; visceral hypersensitivity; gastrointestinal peptides

Irritable bowel syndome (IBS) is a common disorder ${ }^{12}$ but despite this the pathophysiology is far from understood. Visceral hypersensitivity, expressed as lowered perception thresholds to balloon distension in different parts of the gastrointestinal tract ${ }^{3-5}$ and to physiological intestinal motility, ${ }^{6}$ is a feature in many patients. Furthermore, they also demonstrate an altered viscerosomatic referral pattern as another indicator of disturbed processing of viscerosensory information. ${ }^{7}$

Eating a meal stimulates colonic myoelectrical and motor activity. ${ }^{8}$ This gastrocolonic response consists of a gastric phase mediated by mechanoreceptors and an intestinal phase mainly due to stimulation of chemoreceptors where the fat component of the food is the main stimulus. ${ }^{10}{ }^{11}$ Postprandial symptoms are common in IBS $^{12}$ and several studies show a prolonged and exaggerated gastrocolonic response in terms of myoelectrical and motor activity ${ }^{13-15}$ but colonic tone is not altered. ${ }^{16}{ }^{17}$ Recent investigations have shown increased jejunal sensitivity after feeding in both IBS patients and healthy controls ${ }^{18}$ and increased rectal sensitivity after a meal in healthy individuals. ${ }^{19}$ Fat is also the main stimulus for this sensory component of the gastrocolonic response. ${ }^{20}$

The role of gastrointestinal neuropeptides in the generation of symptoms in IBS patients is unclear but differences compared with the normal population have been reported in blood levels of 5-hydroxytryptamine (5-HT), ${ }^{21}$ motilin, cholecystokinin (CCK), ${ }^{22}$ gastrin, and pancreatic polypeptide (PP) ${ }^{23}$ Furthermore, CCK and a high fat meal can unmask dysmotility and produce symptoms in IBS patients. ${ }^{24}$

Most of the previous studies on viscerosensory abnormalities in IBS patients were done in the fasting state and colonic perception thresholds following nutrients have not been investigated previously. As postprandial symptoms are frequent, the aim of the present study was to investigate the sensory component of the gastrocolonic response to a nutrient and to relate the findings to changes in gastrointestinal peptides.

\section{Methods}

SUBJECTS

We studied 16 patients with IBS (14 women, two men; mean age 39 years (range 22-56)) eight with diarrhoea predominant (IBS-D) and eight with constipation predominant (IBS-C)

Abbreviations used in this paper: IBS, irritable bowel syndrome; IBS-D, diarrhoea predominant irritable bowel syndrome; IBS-C, constipation predominant irritable bowel syndrome; IOP, intraoperative pressure; CCK, cholecystokinin; NPY, neuropeptide Y; PYY, peptide YY; PP, pancreatic polypeptide; CRF, corticotrophin releasing factor; 5-HT, 5-hydroxytryptamine; PVEs, phasic volume events; SP, substance P; VIP, vasoactive intestinal polypeptide. 


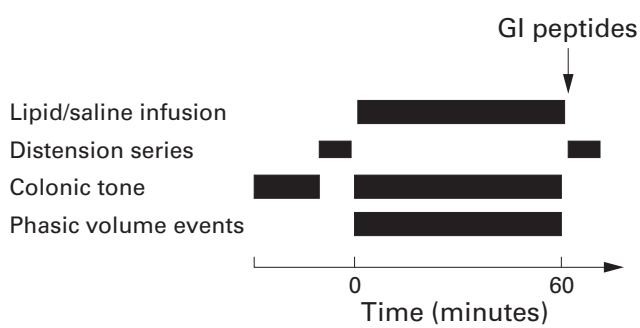

Figure 1 Study design (see text for details).

IBS - referred to our clinic from primary care physicians. All fulfilled the Rome criteria for IBS $^{25}$ and organic gastrointestinal disorders were excluded by routine laboratory tests and colonoscopy with biopsies. The control group consisted of 13 healthy individuals (10 women, three men; mean age 30 years (range 20-43)). Mean age in the control group was significantly lower than that in the patient group $(p=0.02)$. All control subjects were free of gastrointestinal symptoms and had no evidence of acute or chronic illnesses. None was taking any medication known to affect the gastrointestinal tract. All subjects gave informed consent and the study was approved by the ethics committee of the University of Göteborg.

\section{STUDY DESIGN}

The studies took place on two separate days: subjects were randomised to receive a lipid solution (Calogen $1.5 \mathrm{kcal} / \mathrm{ml}$; Nutricia Nordica, Stockholm, Sweden) via an enteral feeding tube (Meda Polyuretansond; Fresenius AG, Bad Homburg, Germany) on one day and $0.9 \%$ saline on the other. Subjects were blinded to the solution they received. The infusion rate was $2 \mathrm{ml} / \mathrm{min}$ and the duration was one hour (fig 1).

After an overnight fast the subjects took a tap water enema $(1500 \mathrm{ml})$ at home and arrived at hospital at 0730 . Under fluoroscopic guidance the enteral feeding tube was placed with its tip in the descending part of the duodenum. The tube was then secured with tape to the cheek. Thereafter the balloon catheter, consisting of a highly compliant balloon made by polyethylene attached to a double lumen polyvinyl tube (Salem Sump Tube, 18F; Sherwood Medical, Tullamore, Ireland), was placed in the mid sigmoid colon. The balloon was tied at both proximal and distal ends and the distance between the attachment sites was $10 \mathrm{~cm}$. Distension to a maximal volume of $550 \mathrm{ml}$ resulted in a cylindrical balloon. A thin thread was inserted through the biopsy channel of a flexible fibreoptic sigmoidoscope and tied to the distal end of the balloon catheter. With the subject in the left lateral position the balloon catheter was carried along the sigmoidoscope to a level of $30-40 \mathrm{~cm}$ from the anal verge. In none of the subjects was retained formed stools observed in the rectum or sigmoid colon during this procedure. The balloon catheter was left in place as the sigmoidoscope was removed. After this procedure the balloon catheter was connected to a computer driven electronic barostat (Dual Drive Barostat, Distender Series II; G\&J Electronics Inc.,
Toronto, Canada). The variation in time for placing the enteral feeding tube and the balloon catheter was small ( $<10$ minutes), and so the experiment was started at the same time of the day in all subjects, approximately 0830 . A small number of distensions up to $25 \mathrm{~mm} \mathrm{Hg}$ were performed to unfold the balloon. Intraoperative pressure (IOP) was set to $2 \mathrm{~mm} \mathrm{Hg}$ above the minimal distending pressure necessary to record respiratory variations in balloon volume. Thereafter a 30 minute equilibrium period was allowed with the balloon pressure at IOP during which colonic tone was assessed (fig 1). Two series of distensions were performed each day - one before and one immediately after administration of lipid or saline. When subjects received the infusions the balloon pressure was set at IOP. Subjects were told to report if they perceived any symptoms during the infusions. Immediately after the end of the infusions venous blood samples were obtained for analyses of gastrointestinal peptides before the second distension series (fig 1).

\section{DISTENSIONS}

We used phasic distensions of 30 seconds' duration starting at IOP and increasing stepwise by $3 \mathrm{~mm} \mathrm{Hg}$ until the subject reported pain or when a pressure of $50 \mathrm{~mm} \mathrm{Hg}$ was reached. Pressure steps were used according to recent international recommendations. ${ }^{26}{ }^{27}$ The distensions were separated by resting periods of 30 seconds with balloon pressure set to IOP. Subjects were instructed to grade their sensations during distensions using a keypad linked to the main barostat. A grading scale consisting of seven parameters was used: 1 , no sensation; 2, fullness; 3, gas; 4, discomfort; 5 , pain; 6 , severe pain; and 7, intolerable pain. A tracking technique (single random staircase) was used, ${ }^{26}$ with tracking beginning when pain $(=5)$ was first reported, after which 10 more distensions were carried out, provided the subject reported pain for at least three of them. Subjects were also instructed to mark the location of their respective sensation on a body map to evaluate the viscerosomatic referral pattern. This was done separately for each distension series.

\section{GASTROINTESTINAL PEPTIDES}

Blood samples were obtained for analysis of plasma levels of motilin, vasoactive intestinal polypeptide (VIP), PP, peptide YY (PYY), CCK, neuropeptide Y (NPY), substance P (SP), and corticotrophin releasing factor (CRF). The samples were immediately centrifuged at $3800 \mathrm{~g}$ at $4^{\circ} \mathrm{C}$ for 10 minutes. The supernatant was then aspirated and stored at $-20^{\circ} \mathrm{C}$ until analysis of the peptides by a radioimmunoassay technique performed at the Department of Neurochemistry, Sahlgrenska University Hospital. CCK could only be analysed in the interval $0.4-12.5 \mathrm{pmol} / 1$.

\section{DATA ANALYSIS}

The Protocol Plus software package (G\&J Electronics Inc., Toronto, Canada) was used for data analysis. The pain threshold was the average pressure of the distensions where pain was reported. If pain was not experienced, the 
Table 1 Baseline thresholds in the control (C) and irritable bowel syndrome (IBS) groups

\begin{tabular}{lllll}
\hline & $C(n=13)$ & $I B S(n=16)$ & $I B S-C(n=8)$ & $I B S-D(n=8)$ \\
\hline First sensation & $26.4(2.5)$ & $20.5(1.4)^{\star}$ & $21.2(1.9)$ & $19.8(2.0)$ \\
Gas & $36.4(3.0)$ & $26.7(1.3)^{\star \star}$ & $27.2(1.4)^{\star}$ & $26.2(2.1)^{\star}$ \\
Discomfort & $43.3(2.3)$ & $37.0(1.7)^{\star}$ & $38.1(2.7)$ & $35.9(2.2)^{\star}$ \\
Pain & $46.4(1.5)$ & $43.3(1.7)$ & $45.0(1.9)$ & $41.7(2.8)$ \\
\hline
\end{tabular}

Baseline thresholds ( $\mathrm{mm} \mathrm{Hg}$ ) - that is, before infusions-in the IBS group (IBS) and in the subgroups with predominant constipation (IBS-C) and diarrhoea (IBS-D) are shown. The thresholds are an average of the two study days.

${ }^{\star} \mathrm{p}<0.05 ;{ }^{\star \star} \mathrm{p}<0.01$ compared with controls.

pain threshold was set to a maximum pressure of $50 \mathrm{~mm} \mathrm{Hg}$. The thresholds for first sensation, gas, discomfort, and pain before compared with after saline and lipid, respectively, were compared in each group (healthy controls, IBS, IBS-D, IBS-C). Baseline thresholds - that is, before enteral administration of the solution - and the percent reduction in thresholds after lipids and saline were compared between groups. Colonic balloon volumes, reflecting tone, were assessed with pressure set at IOP. Barostat balloon volumes were averaged over 10 minutes and mean volumes were calculated for 30 minute periods before distensions (fasting tone) and during the first and second 30 minutes of the infusions, respectively. Balloon volumes before compared with during administration of the solutions were then compared. In addition, the number of phasic volume events (PVEs), defined as changes of $\geqslant 10 \%$ compared with baseline volume and occurring at a rate of $1-4 /$ minute, ${ }^{28}$ were calculated during the infusions and compared between groups and the two study days (fig 1). Compliance curves, that is pressurevolume relationship, were also created for each distension series by plotting the volume increase $(\Delta \mathrm{V})$ against the corresponding pressure level above IOP $(\Delta \mathrm{P})$. This was done up to the pressure level which the majority of subjects reached during the distension series both before and after the infusions. Changes within and between groups were then analysed. To assess the viscerosomatic referral pattern we calculated the relative area of referred discomfort on the body maps. The results were then compared before versus after the infusions and also between groups. Plasma levels of gastrointestinal peptides after lipid infusion compared with saline were assessed for each group and differences between groups analysed.

\section{STATISTICS}

Results are expressed as mean (SEM) with the exception of the gastrointestinal peptides, where median and interquartile range are used because highly skewed data were obtained for some of the peptides. Non-parametric tests were used for analysis of the peptides. The compliance curves were compared using analysis of variance (ANOVA), and the $\chi^{2}$ test with continuity correction was used for comparisons of symptoms during the infusions. Otherwise the $t$ test was used for statistical comparisons. Significance was accepted at the 0.05 level.

\section{Results}

PERCEPTION THRESHOLDS BEFORE INFUSIONS As shown in table 1, the IBS group exhibited lower baseline thresholds than the control subjects for first sensation, gas, and discomfort but the pain threshold did not differ between groups $(p=0.18)$. Compared with the control group, lower baseline thresholds were observed in the IBS-D group for gas and discomfort and in the IBS-C group only for gas (table 1). The baseline thresholds did not differ between the IBS-D and IBS-C groups ( $p>0.20$ for all sensations). The perception thresholds before infusion did not differ between the study days (data not shown).

PERCEPTION THRESHOLDS AFTER INFUSION The lipid infusion was generally well tolerated. However, six controls and seven IBS patients developed mild nausea ( $p>0.20)$, seven controls and 12 IBS patients experienced mild abdominal distension ( $p>0.20)$, and urgency was felt by four control subjects and eight IBS patients $(p>0.20)$. After saline, patients exhibited a small reduction in pain threshold compared with baseline $(41.8$ (2.3) $\mathrm{mm} \mathrm{Hg} v$ 39.9 (2.7) $\mathrm{mm} \mathrm{Hg}$; =0.006). Otherwise there were no changes after saline infusion (data not shown). After lipid infusion, control subjects demonstrated lowered thresholds compared with baseline for gas and trends in the same direction for discomfort and pain (fig 2A). Volumes at the perception thresholds were also reduced in controls after lipid infusion compared with baseline, although this was not sig-
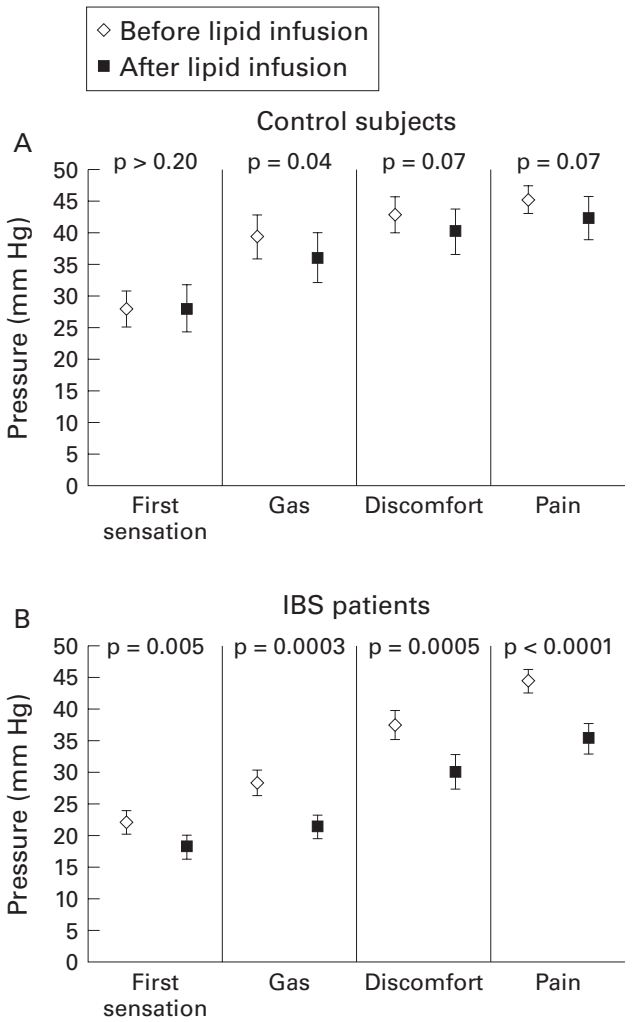

Figure 2 Perception thresholds (mean (SEM)) before and after lipid infusion in the control group $(A)$ and irritable bowel syndrome (IBS) group (B). p values refer to comparisons of thresholds before versus after lipid infusion within groups. 


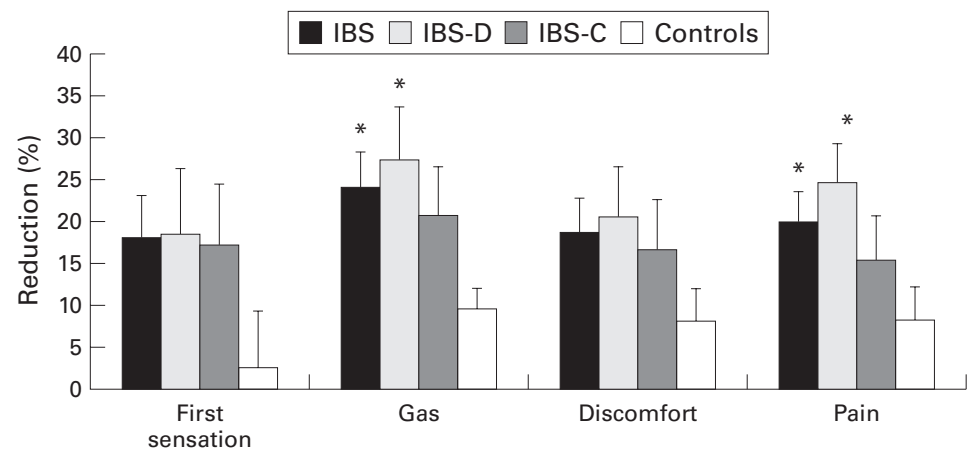

Figure 3 Comparisons of reduction in perception thresholds showed greater reduction for gas $(p=0.03)$ and pain $(p=0.04)$ in irritable bowel syndrome (IBS) patients compared with controls, and tendencies to lowered thresholds for first sensation $(p=0.08)$ and

discomfort $(p=0.08)$. For the IBS subgroups the reduction was greater in the IBS-D group than in controls for gas $(p=0.03)$ and pain $(p=0.02)$ but between IBS-C and controls no significant differences were observed. ${ }^{\star} p<0.05$ compared with controls.

nificant for gas (212 (29) v 203 (29) $\mathrm{ml}$; $\mathrm{p}=0.16)$, discomfort $(230$ (15) v 220 (27) $\mathrm{ml}$; $\mathrm{p}=0.06$ ), or pain (251 (25) v 230 (26) $\mathrm{ml}$; $\mathrm{p}=0.06)$. Highly significant reductions in perception thresholds for all sensations compared with baseline were observed after lipid infusion in IBS patients (fig 2B). Also, volumes at the perception thresholds were significantly reduced for all sensations after lipid infusion compared with baseline in IBS patients-that is, for first sensation (159 (12) v 124 (13) $\mathrm{ml}$; $\mathrm{p}=0.05)$, gas $(186(15) v 142$ (13) $\mathrm{ml} ; \mathrm{p}=0.01)$, discomfort (230 (17) v 202 (17) $\mathrm{ml} ; \mathrm{p}=0.005)$, and pain (264 (19) $v 230$ (17) $\mathrm{ml} ; \mathrm{p}=0.003)$.

The thresholds in the IBS subgroups were also evaluated. After lipid infusion, IBS-C patients had lowered perception thresholds for gas (29.8 (2.4) mm Hg v 23.5 (2.5) $\mathrm{mm} \mathrm{Hg}$; $\mathrm{p}=0.01)$, discomfort $(41.9(2.6) \mathrm{mm} \mathrm{Hg} v 35.4$ (4.0) $\mathrm{mm} \mathrm{Hg}$; $=0.03$ ), and pain (47.6 (1.5) $\mathrm{mm} \mathrm{Hg} v 40.5$ (3.2) $\mathrm{mm} \mathrm{Hg}$; $=0.02$ ) and the same was also true for the IBS-D group (gas: 26.6 (3.3) $\mathrm{mm} \mathrm{Hg} v 19.0$ (2.4) $\mathrm{mm} \mathrm{Hg}$ $(\mathrm{p}=0.02)$; discomfort: 32.9 (3.0) $\mathrm{mm} \mathrm{Hg} v$ 24.5 (2.5) $\mathrm{mm} \mathrm{Hg}(\mathrm{p}=0.01)$; and pain: 41.1 (3.2) $\mathrm{mm} \mathrm{Hg} v 29.8$ (2.7) $\mathrm{mm} \mathrm{Hg}(\mathrm{p}=0.002))$.

Comparisons between patients and controls (percent reduction in thresholds) revealed that the reduced thresholds for gas and pain in IBS patients were significantly greater and trends in the same direction were observed for the two other thresholds studied. The IBS-D group differed in the same way from controls but that was not the case for the IBS-C group whose reduced thresholds did not differ significantly from control subjects (fig 3). We also analysed how many subjects in each group had reduced perception thresholds $\geqslant 10 \%$ after lipid infusion. For gas and pain it was 13/16 IBS patients compared with $4 / 13$ control subjects $(p=0.02)$, and for discomfort 12/16 v 5/13 ( $\mathrm{p}=0.11)$.

None of the subjects reported "intolerable pain" during distensions and a minority reported "severe pain"; hence these descriptors were not used for further analysis.

COLONIC TONE AND COMPLIANCE

The minimal distending pressure did not differ between patients and controls (9.8 (0.8) $\mathrm{mm} \mathrm{Hg} v 9.7$ (0.6) $\mathrm{mm} \mathrm{Hg}$; $>0.20)$. No changes in colonic balloon volumes were

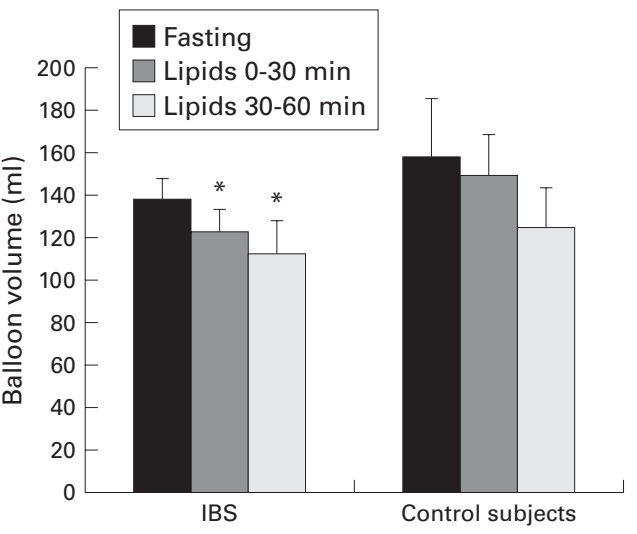

Figure 4 Barostat balloon volumes, reflecting colonic tone, in the fasting state and during the first and second 30 minutes of the lipid infusion in the irritable bowel syndrome (IBS) and control groups. The reductions in balloon volumes during infusion compared with the fasting state in the control group did not reach statistical significance ( $p>0.20$ for $0-30$ minutes; $p=0.10$ for $30-60$ minutes); ${ }^{*} p<0.05$ compared with the fasting recording.

observed during the saline experiment and there were no significant differences between groups (data not shown). In four control subjects and one patient, adequate tone recordings were not obtained during lipid infusion due to technical problems and the results of these subjects were excluded. During lipid infusion balloon volumes decreased in both groups compared with the fasting recording, reflecting increased colonic tone, both during the first and second 30 minutes of the infusions (fig 4). However, only the reduction in the patient group reached statistical significance. No significant differences in tone between patients and controls were observed in the fasting state or during infusions $(p>0.20$ for all comparisons). There was no correlation between the increase in colonic tone and percent reduction in perception thresholds $(p>0.20$ for all sensations). The number of PVEs was not significantly different during the lipid infusion compared with the saline infusion in patients (31 (5) v 27 (6); $\mathrm{p}>0.20$ ) or controls (16 (5) $v$ 9 (3); $p=0.18)$. The IBS group demonstrated more PVEs during both saline $(p=0.01)$ and lipid $(p=0.04)$ infusions compared with controls. The infusions did not produce any significant changes in compliance (fig 5). In control subjects however, a trend towards higher compliance after saline $(p=0.11)$ and after lipid $(p=0.15)$ infusions was noted but no such trend was seen in patients $(p>0.20)$. Compliance tended to be higher in patients but no significant differences were observed.

\section{THE VISCEROSOMATIC REFERRAL PATTERN}

In the patient group the relative area of referred discomfort increased after lipids compared with baseline $\left(15.2(3.2) \mathrm{cm}^{2} v 9.0(2.0) \mathrm{cm}^{2}\right.$; $\mathrm{p}=0.01)$. The same was also seen for the relative area of referred pain in patients $(15.2$ (3.5) $\mathrm{cm}^{2} v 8.2$ (3.2) $\left.\mathrm{cm}^{2} ; \mathrm{p}=0.03\right)$. In control subjects, the relative area did not change significantly after lipid infusion compared with baseline for discomfort $\left(4.9\right.$ (1.2) $\mathrm{cm}^{2} v 5.6$ (1.3) $\left.\mathrm{cm}^{2} ; \mathrm{p}=0.15\right)$ or pain $\left(6.8(1.7) \mathrm{cm}^{2} v 5.7\right.$ (2.1) $\left.\mathrm{cm}^{2} ; \mathrm{p}>0.20\right)$. In the IBS group, $69 \%$ 

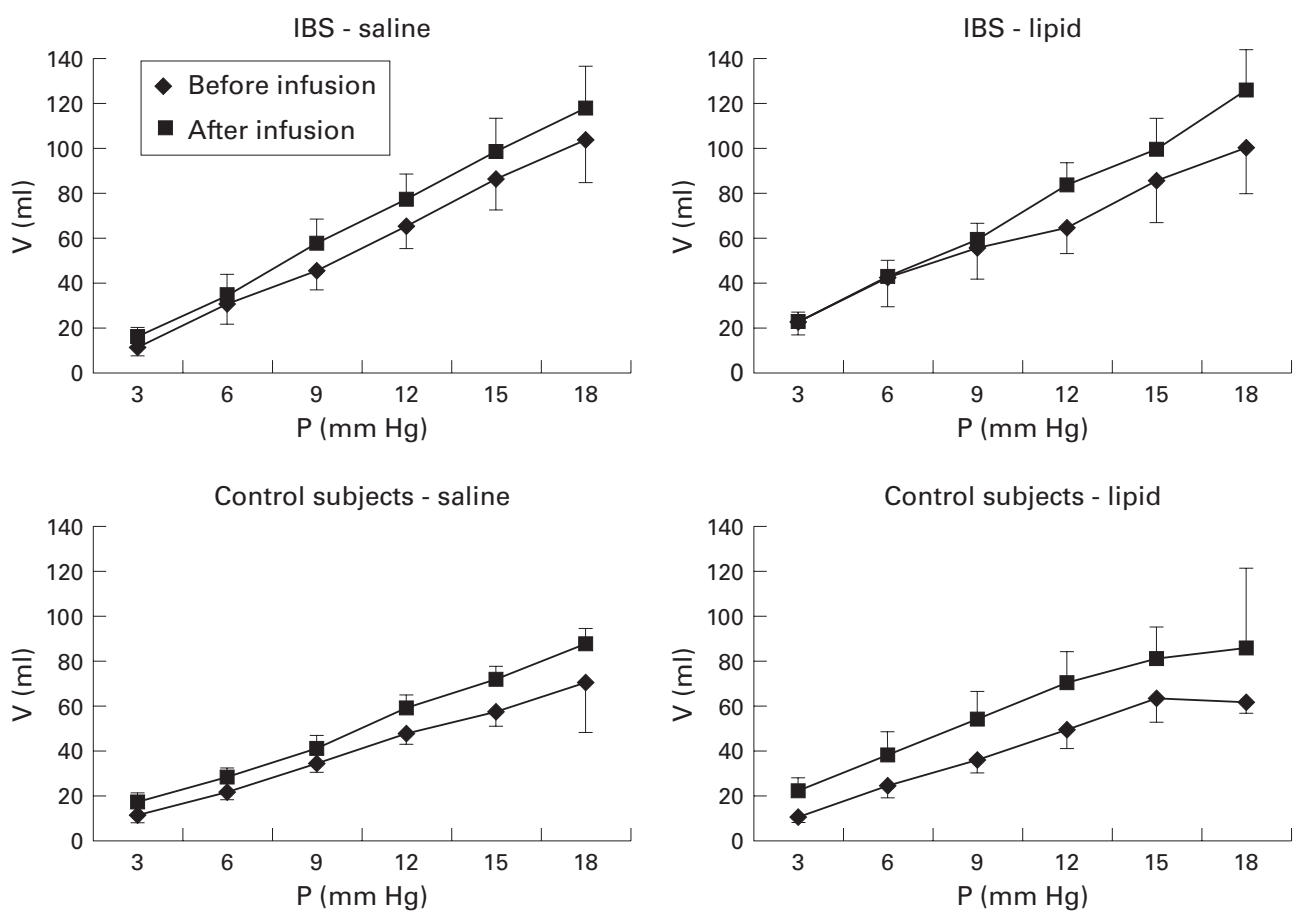

Figure 5 Colonic compliance curves for the irritable bowel syndrome (IBS) and control groups before and after saline and lipid, respectively, shown as volume increase (V) for corresponding pressure increase $(P)$ above intraoperative pressure (IOP).

$(11 / 16)$ of subjects had increased abdominal and back areas of referred discomfort after lipids compared with baseline (fig 6) whereas this was seen in only $8 \%(1 / 13)$ of the control group $(\mathrm{p}<0.01)$. After saline, no changes in viscerosomatic referral patterns compared with baseline were seen.

GASTROINTESTINAL PEPTIDES

Higher levels of CCK, PP, PYY, VIP, and NPY were seen after lipid compared with after saline

IBS: Viscerosomatic referral pattern

A Before lipids
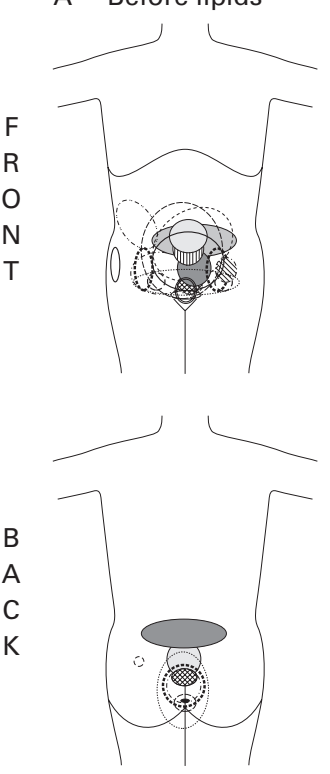

Figure 6 Abdominal and back areas of referred discomfort before and after lipid infusion in the irritable bowel syndrome (IBS) patients, reflecting the viscerosomatic referral pattern. Each patient has its own pattern, but can have more than one circle. infusion in both the IBS group and the control group, but SP and CRF levels did not differ (table 2). Higher motilin levels after saline infusion in the patient groups were seen $(p=0.04)$ and a tendency towards higher levels after lipid infusion $(p=0.08)$. In the IBS group several very high motilin levels were seen, particularly in the IBS-C group. IBS-C patients exhibited higher motilin levels after saline than after lipids (116 (68-147) $v 102$ (56-136) $\mathrm{pmol} / \mathrm{l}$ (median (IQR)); $\mathrm{p}=0.02$ ) while a trend in the opposite direction was noted for IBS-D patients $(70(61-85)$ v $76(70-115) \mathrm{pmol} / \mathrm{l}$; $\mathrm{p}=0.06)$. Levels of SP tended to be higher in the control group than in the IBS group after lipids $(p=0.05)$. Lower CRF levels were seen in the IBS-C group compared with the IBS-D group after saline (40.5 (35.0-41.5) v 45.0 (42.5-47.8) $\mathrm{pmol} / \mathrm{l} ; \mathrm{p}=0.02)$ and lipids (39.0 $(34.5-41.0) \quad v \quad 44.5 \quad(43.0-48.0) \quad \mathrm{pmol} / 1$; $\mathrm{p}=0.005)$. There were no other differences between groups.

\section{Discussion}

We have demonstrated an exaggerated sensory component of the gastrocolonic response in IBS patients, expressed as a marked reduction in colonic perception thresholds and alteration of the viscerosomatic referral pattern after lipid administration in the upper gut. Together with postprandial motor alterations seen in IBS, ${ }^{13-15}$ our findings may explain postprandial symptoms in IBS patients.

As in many other studies, IBS patients in our study demonstrated visceral hypersensitivity compared with healthy individuals in the fasting state. ${ }^{329}$ However, pain thresholds did not differ between groups but this may have been for methodological reasons. A significant number of subjects did not experience pain at 
Table 2 Gastrointestinal peptides in the control and irritable bowel syndrome (IBS) groups

\begin{tabular}{|c|c|c|c|c|}
\hline & \multicolumn{2}{|l|}{ Control subjects } & \multicolumn{2}{|l|}{ IBS } \\
\hline & Saline & Lipid & Saline & Lipid \\
\hline CCK & $<0.4(<0.4-0.7)$ & $>12.5^{\star \star}(4.7->12.5)$ & $0.7(<0.4-9.6)$ & $11.4^{\star}(2.7->12.5)$ \\
\hline Motilin & $65 \quad(50-76)$ & $73(61-78)$ & $79 \quad(63-131)$ & $79(63-122)$ \\
\hline PP & $22 \quad(15-35)$ & $57^{\star \star}(24-74)$ & $(15-33)$ & $37^{\star}(23-71)$ \\
\hline PYY & $20 \quad(19-23)$ & $30^{\star \star}(28-34)$ & $23 \quad(16-25)$ & $30^{\star \star}(27-36)$ \\
\hline VIP & $8.0(7.0-11.2)$ & $11.0^{\star \star}(8.8-13.8)$ & $7.0(5.0-9.8)$ & $11.0^{\star}(8.0-13.5)$ \\
\hline NPY & $108 \quad(94-121)$ & $117^{\star \star}(104-132)$ & $105 \quad(97-110)$ & $118^{\star \star}(106-127)$ \\
\hline SP & $1.4(1.2-1.8)$ & $1.7(1.4-2.1)$ & $1.3(1.1-1.5)$ & $1.4(1.0-1.7)$ \\
\hline CRF & $42 \quad(38-48)$ & $41(39-48)$ & $42 \quad(39-45)$ & $42(39-44)$ \\
\hline
\end{tabular}

Plasma levels of gastrointestinal peptides ( $\mathrm{pmol} / \mathrm{l}$; median, interquartile range (IQR)). ${ }^{\star} \mathrm{p}<0.05,{ }^{\star \star} \mathrm{p}<0.01$, plasma levels after lipid compared with after saline.

CCK, cholecystokinin; PP, pancreatic polypeptide; PYY, peptide YY; VIP, vasoactive intestinal polypeptide; NPY, neuropeptide Y; SP, substance P; CRF, corticotrophin releasing factor.

baseline up to $50 \mathrm{~mm} \mathrm{Hg}$ but their pain threshold was set to $50 \mathrm{~mm} \mathrm{Hg}$, which is an underestimation, and may have influenced the statistical comparison of pain thresholds between groups.

In our study, IBS patients were slightly older than controls. The small age difference between groups is unlikely to explain the differences in baseline thresholds between groups as recent studies have shown decreased rectal sensitivity with increasing age. ${ }^{27} 30$

After saline infusion the only difference compared with baseline was a small reduction in pain threshold in the patient group. We consider three possible explanations for this observation. Despite using a tracking technique to minimise psychological influences on the results, ${ }^{26}$ it is possible that the reduced pain threshold was due to psychological bias. Another possible explanation is that the first distension series, with several painful distensions, caused central sensitisation resulting in lower pain thresholds in the following series of distensions. ${ }^{31} 32$ There are conflicting results regarding the reproducibility of sensory thresholds determined on the same day. ${ }^{33} 34$ Our results, with no threshold changes in healthy controls during the saline experiment, indicate good reproducibility of the threshold determinations in our study. Therefore, we believe that the first two mechanisms are more likely explanations of the reduced pain threshold in patients after saline. However, these cannot explain the observed reduction in perception thresholds following lipid administration as this reduction was of a greater magnitude, particularly in the IBS group.

The small but significant reduction in the threshold for gas following lipid infusion in the control group and the tendency to lower thresholds for discomfort and pain are in agreement with previous reports in healthy controls. ${ }^{19}{ }^{20}$ There seems to be a sensory component to the gastrocolonic response to food in healthy individuals although in the current study it was rather modest and limited to a small number of individuals. In the IBS group the reduction was more pronounced and a more constant phenomenon as the majority of patients demonstrated reduced thresholds after lipids. Both IBS subgroups had lowered thresholds after lipids but only in the IBS-D group was the reduction clearly different from controls. These findings are in agreement with another study showing differences in subgroups of IBS patients. ${ }^{35}$ However, the relatively small numbers of subjects in the subgroups may explain the lack of significant changes between the IBS-C group and controls (type II error).

Tonic and phasic motor activity was evaluated by measuring colonic balloon volumes (tone) and phasic volume events ${ }^{28}$ during and before infusions. Colonic balloon volumes were reduced by about $20 \%$ in both groups during lipid infusion, reflecting increased tone. This is comparable with results obtained by Ford and colleagues. ${ }^{36}$ However, due to higher variability and a smaller number of subjects in the control group, the reduction in volume was significant only in patients. No differences in tone between groups were observed and no correlation between the increase in colonic tone and reduction in sensory thresholds was demonstrated. Thus it is unlikely that the pronounced reduction in perception thresholds after lipids in patients was solely secondary to the tonic motor response. Furthermore, in previous studies neither fasting nor postprandial colonic tone was found to be different in IBS patients compared with healthy controls. ${ }^{16}{ }^{17}$ The phasic motor events were more frequent in patients during both the saline and lipid experiments but there were no differences between saline and lipids. Therefore, increased phasic motor activity is not a likely explanation for the observed group differences in perception after lipids compared with saline.

The viscerosomatic referral pattern in IBS patients differs from that in healthy individuals ${ }^{7}$ and a conditioning stimulus in the form of repetitive noxious sigmoid stimulation can increase the referral area of sensations provoked by rectal distension in IBS patients. ${ }^{32}$ These findings are thought to reflect abnormal spinal processing of viscerosensory information. ${ }^{37}$ In our study IBS patients, but not control subjects, had an increased area of referred discomfort and pain following lipid infusion. This may be due to central sensitisation of the dorsal horn neurones. ${ }^{32}$ This sensitisation may be secondary to an exaggerated postprandial motor response in IBS patients yielding more high amplitude contractions. ${ }^{15-17}$

The pattern of increased levels of several gastrointestinal peptides after lipid infusion has previously been reported for CCK, ${ }^{38} \mathrm{PYY}^{39}$ and $\mathrm{PP}^{40}$ The increase was similar in controls and patients. Therefore, our peptide data do not explain the pronounced threshold reduction in IBS patients. However, altered sensitivity to some of the peptides in the IBS group may be one explanation. It has previously been shown that infusion of cholecystokinin octapeptide (CCK-8) can provoke abnormal reactions of the gall bladder in IBS patients ${ }^{41}$ and higher pain scores in patients with functional abdominal pain, ${ }^{42}$ and unmask dysmotility in IBS patients. ${ }^{24}$

Motilin, known to act primarily in the upper gut $^{43}$ but also proposed to stimulate colonic motor activity, ${ }^{44}$ and CRF were the only peptides that differed between groups. Higher motilin levels were observed in patients, but 
more interesting were levels of motilin in the IBS subgroups. Preston et al have shown impaired motilin release after drinking water in constipated patients and related these findings to disturbances in transit time, ${ }^{23}$ and patients with IBS-C have prolonged, and IBS-D shorter, whole gut transit time. ${ }^{45}$ These findings are to some extent in accordance with ours, as the group where slow transit is more likely (IBS-C) had lowered motilin levels after lipid infusion whereas the opposite was seen in IBS-D patients - that is, patients with probable accelerated transit time. The lower CRF levels in the constipated patients compared with the diarrhoea group were also interesting as CRF has been shown to stimulate colonic motor function. ${ }^{46}$ However, one must be cautious when interpreting our peptide data as no release profile of the peptides was analysed which is probably a more relevant approach.

There are several possible mechanisms for the pronounced reduction in thresholds in the IBS group. Reports of higher postprandial 5-HT levels in IBS patients with diarrhoea compared with healthy individuals ${ }^{21}$ and promising results in the treatment of patients with IBS with $5-\mathrm{HT}_{3}$ antagonists ${ }^{47}$ indicate that 5-HT may be a mediator of the sensory component. Another possibility is that psychological factors could have influenced the lowered thresholds ${ }^{48}$ as the symptoms experienced during lipid infusion could have triggered a stress response, particularly in IBS patients. The altered viscerosomatic referral pattern, demonstrated in patients, is thought to reflect abnormal spinal processing of viscerosensory information ${ }^{37}$ and psychological factors may have influenced this through descending inhibitory and facilitatory pathways. However, some patients did not experience any symptoms during lipid infusion but still had an altered viscerosomatic referral pattern and reduced perception thresholds after lipid infusion. In our opinion, this argues against the importance of psychological factors for our findings.

In conclusion, we have demonstrated that IBS patients exhibit an exaggerated sensory component of the gastrocolonic response, expressed as lowered colonic perception thresholds and increased viscerosomatic referral area after duodenal lipid infusion. This may be an important factor in the postprandial symptoms in IBS patients. However, the mechanism remains to be clarified.

This study was supported by the Swedish Medical Research Council (grants 8288 and 13409) and by the Faculty of Medi-
cine, University of Göteborg. The authors would like to express cine, University of Göteborg. The authors would like to express ance during the experiments.

1 Longstreth GF, Wolde-Tsadik G. Irritable bowel-type symptoms in HMO examinees. Prevalence, demographics, and clinical correlates. Dig Dis Sci 1993;38:1581-9.

2 Agreus L, Svärdsudd K, Nyren O, et al. Irritable bowel syndrome and dyspepsia in the general population: overlap and lack of stability over time. Gastroenterology 1995;109: and lack

3 Ritchie J. Pain from distension of the pelvic colon by inflating a balloon in the irritable colon syndrome. Gut 1973;14: ing a balloo $125-32$.

4 Accarino AM, Azpiroz F, Malagelada JR. Selective dysfunction of mechanosensitive intestinal afferents in irritable bowel syndrome. Gastroenterology 1995;108:636-43.
5 Costantini M, Sturniolo GC, Zaninotto G, et al. Altered esophageal pain threshold in irritable bowel syndrome. Dig Dis Sci 1993;38:206-12.

6 Kellow JE, Eckersley CM, Jones MP. Enhanced perception of physiological intestinal motility in the irritable bowel syndrome. Gastroenterology 1991;101:1621-7.

7 Swarbrick ET, Hegarty JE, Bat L, et al. Site of pain from the irritable bowel. Lancet 1980;2:443-6.

8 Snape WJ Jr, Matarazzo SA, Cohen S. Effect of eating and gastrointestinal hormones on human colonic myoelectrical and motor activity. Gastroenterology 1978;75:373-8.

9 Steadman CJ, Phillips SF, Camilleri M, et al. Variation of muscle tone in the human colon. Gastroenterology 1991; 101:373-81.

10 Wiley J, Tatum D, Keinath R, et al. Participation of gastric mechanoreceptors and intestinal chemoreceptors in the gastrocolonic response. Gastroenterology 1988;94:1144-9.

11 Wright SH, Snape WJ Jr, Battle W, et al. Effect of dietary components on gastrocolonic response. Am F Physiol 1980; 238:G228-32.

12 Ragnarsson G, Bodemar G. Pain is temporally related to eating but not to defaecation in the irritable bowel syndrome (IBS). Patients' description of diarrhea, constipation and symptom variation during a prospective 6-week study. Eur 7 Gastroenterol Hepatol 1998;10:415-21.

13 Sullivan MA, Cohen S, Snape WJ Jr. Colonic myoelectrical activity in irritable-bowel syndrome. Effect of eating and activity in irritable-bowel syndrome. Effect of eatin

14 Narducci F, Bassotti G, Granata MT, et al. Colonic motility and gastric emptying in patients with irritable bowel syndrome. Effect of pretreatment with octylonium bromide. Dig Dis Sci 1986;31:241-6.

5 Rogers J, Henry MM, Misiewicz JJ. Increased segmental activity and intraluminal pressures in the sigmoid colon of patients with the irritable bowel syndrome. Gut 1989;30: 634-41.

16 Vassallo MJ, Camilleri M, Phillips SF, et al. Colonic tone and motility in patients with irritable bowel syndrome. Mayo Clin Proc 1992;67:725-31.

17 Björnsson ES, Chey WD, Hooper F, et al. Selectively enhanced phasic but not tonic gastro-colonic contractions in diarrhea-predominant irritable bowel syndrome: Role of 5-HT3 neural pathways. Gastroenterology 1998;114:A722.

18 Evans PR, Kellow JE. Physiological modulation of jejunal sensitivity in health and in irritable bowel syndrome. Am $\mathcal{F}$ Gastroenterol 1998;93:2191-6.

19 Musial F, Crowell MD, Kalveram KT, et al. Nutrient ingestion increases rectal sensitivity in humans. Physiol Behav 1994;55:953-6.

20 Erckenbrecht JF, Hemstege M, Rühl A, et al. The sensory component of the gastrocolonic response revisited: Postprandial colonic perception depends on meal composition. Gastroenterology 1994;106:A494.

21 Bearcroft CP, Perrett D, Farthing MJ. Postprandial plasma 5-hydroxytryptamine in diarrhoea predominant irritable 5-hydroxytryptamine in diarrhoea predominant

22 Sjölund K, Ekman R, Lindgren S, et al. Disturbed motilin and cholecystokinin release in the irritable bowel syndrome. Scand $\mathcal{F}$ Gastroenterol 1996;31:1110-14.

23 Preston DM, Adrian TE, Christofides ND, et al. Positive correlation between symptoms and circulating motilin, pancreatic polypeptide and gastrin concentrations in functional bowel disorders. Gut 1985;26:1059-64.

24 Kellow JE, Phillips SF, Miller LJ, et al. Dysmotility of the small intestine in irritable bowel syndrome. Gut 1988;29. 1236-43.

25 Thompson WG, Creed F, Drossman DA, et al. Functional bowel disease and functional abdominal pain. Gastroenterol Int 1992;5:75-91.

26 Whitehead WE, Delvaux M. Standardization of barostat procedures for testing smooth muscle tone and sensory thresholds in the gastrointestinal tract. The Working Team of Glaxo-Wellcome Research, UK. Dig Dis Sci 1997;42: $223-41$

27 Lagier E, Delvaux M, Vellas B, et al. Influence of age on rectal tone and sensitivity to distension in healthy subjects. Neurogastroenterol Motil 1999;11:101-7.

28 von der Ohe MR, Hanson RB, Camilleri M. Serotonergic mediation of postprandial colonic tonic and phasic responses in humans. Gut 1994;35:536-41.

29 Bradette M, Delvaux M, Staumont G, et al. Evaluation of colonic sensory thresholds in IBS patients using a barostat. Definition of optimal conditions and comparison with healthy subjects. Dig Dis Sci 1994;39:449-57.

30 Mertz H, Naliboff B, Munakata J, et al. Altered rectal perception is a biological marker of patients with irritable perception is a biological marker of patients with
bowel syndrome. Gastroenterology 1995;109:40-52.

31 Ness TJ, Metcalf AM, Gebhart GF. A psychophysiological study in humans using phasic colonic distension as a noxious visceral stimulus. Pain 1990;43:377-86.

32 Munakata J, Naliboff B, Harraf F, et al. Repetitive sigmoid stimulation induces rectal hyperalgesia in patients with irritable bowel syndrome. Gastroenterology 1997;112:55-63.

33 Lembo T, Munakata J, Mertz H, et al. Evidence for the hypersensitivity of lumbar splanchnic afferents in irritable bowel syndrome. Gastroenterology 1994;107:1686-96.

34 Hammer HF, Phillips SF, Camilleri M, et al. Rectal tone, distensibility, and perception: reproducibility and response
to different distensions. Am $\mathcal{F}$ Physiol 1998;274:G584-90.

35 Prior A, Maxton DG, Whorwell PJ. Anorectal manometry in irritable bowel syndrome: differences between diarrhoea and constipation predominant subjects. Gut 1990;31:45862 . 
36 Ford MJ, Camilleri M, Wiste JA, et al. Differences in colonic tone and phasic response to a meal in the transverse and sigmoid human colon. Gut 1995;37:264-9.

37 Mayer EA, Gebhart GF. Basic and clinical aspects of visceral hyperalgesia. Gastroenterology 1994;107:271-93.

38 Liddle RA, Goldfine ID, Rosen MS, et al. Cholecystokinin bioactivity in human plasma. Molecular forms, responses to feeding, and relationship to gallbladder contraction. $\mathcal{F}$ Clin Invest 1985;75:1144-52.

39 Spiller RC, Trotman IF, Adrian TE, et al. Further Spiller RC, Trotman IF, Adrian TE, et al. Further
characterisation of the 'ileal brake' reflex in man-effect of characterisation of the 'ileal brake' reflex in man-effect of
ileal infusion of partial digests of fat, protein, and starch on jejunal motility and release of neurotensin, enteroglucagon, and peptide YY. Gut 1988;29:1042-51.

40 Isaacs PE, Ladas S, Forgacs IC, et al. Comparison of effects of ingested medium- and long-chain triglyceride on gallbladder volume and release of cholecystokinin and other gut peptides. Dig Dis Sci 1987;32:481-6.

41 Kellow JE, Miller LJ, Phillips SF, et al. Altered sensitivity of the gallbladder to cholecystokinin octapeptide in irritable bowel syndrome. Am F Physiol 1987;253:G650-5.
42 Roberts-Thomson IC, Fettman MJ, Jonsson JR, et al. Responses to cholecystokinin octapeptide in patients with functional abdominal pain syndromes. $f$ Gastroenterol Hepatol 1992;7:293-7.

43 Vantrappen G, Janssens J, Peeters TL, et al. Motilin and the interdigestive migrating motor complex in man. Dig Dis Sci 1979;24:497-500

44 Rennie J, Christofides N, Mitchenere P, et al. Motilin and human colonic activity. Gastroenterology 1980;78:A1243.

45 Cann PA, Read NW, Brown C, et al. Irritable bowel syndrome: relationship of disorders in the transit of a single solid meal to symptom patterns. Gut 1983;24:405-11.

46 Tache Y, Martinez V, Million M, et al. Corticotropinreleasing factor and the brain-gut motor response to stress. Can 7 Gastroenterol 1999;13(suppl A):18-25A.

47 Mangel AW, Northcutt AR. Review article: the safety and efficacy of alosetron, a 5-HT3 receptor antagonist, in female irritable bowel syndrome patients. Aliment Pharmacol Ther 1999;13(suppl 2):77-82.

48 Ford MJ, Camilleri M, Zinsmeister AR, et al. Psychosensory modulation of colonic sensation in the human transverse and sigmoid colon. Gastroenterology 1995;109:1772-80. 